\title{
Twin nucleation in Fe-based bcc alloys-modeling and experiments
}

\author{
A Ojha ${ }^{1}, \mathrm{H}$ Sehitoglu ${ }^{1}$, L Patriarca ${ }^{1}$ and $\mathrm{H} \mathrm{J}$ Maier $^{2}$ \\ ${ }^{1}$ Department of Mechanical Science and Engineering, University of \\ Illinois at Urbana-Champaign, 1206 West Green Street, Urbana, IL 61801, USA \\ ${ }^{2}$ Institut für Werkstoffkunde, Leibniz Universität Hannover, An der Universität 2, \\ D-30823 Garbsen, Germany \\ E-mail: huseyin@illinois.edu
}

Received 30 April 2014, revised 25 July 2014

Accepted for publication 7 August 2014

Published 6 October 2014

\begin{abstract}
We develop an analytical expression for twin nucleation stress in bcc metal and alloys considering generalized planar fault energy and the dislocations bounding the twin nucleus. We minimize the total energy to predict the twinning stress relying only on parameters that are obtained through atomistic calculations, thus excluding the need for any empirical constants. We validate the present approach by means of precise measurements of the onset of twinning in bcc $\mathrm{Fe}-50$ at $\% \mathrm{Cr}$ single crystals showing excellent agreement. The experimental observations of the three activated slip systems of symmetric configuration in relation to the twinning mechanism are demonstrated via transmission electron microscopy techniques along with digital image correlation. We then confirm the validity of the model for $\mathrm{Fe}, \mathrm{Fe}-25 \mathrm{at} \% \mathrm{Ni}$ and $\mathrm{Fe}-3 \mathrm{at} \% \mathrm{~V}$ alloys compared with experiments from the literature to show general applicability.
\end{abstract}

Keywords: bcc, twinning, DIC, molecular dynamics

(Some figures may appear in colour only in the online journal)

\section{Introduction}

A number of technologically important bcc alloys undergo twinning deformation as summarized in the review articles [1-3]. Early works proposed the following twin nucleation mechanisms in bec metals: (i) the pole mechanism proposed by Cottrell and Bilby [4], (ii) the slip dislocation interaction mechanism proposed by Priestner and Leslie [5], (iii) the dislocation core dissociation mechanism suggested by Sleeswyk [6] and Lagerlof [7] and (iv) the edge dislocation dissociation mechanism proposed by Ogawa [8]. We provide the twinning stress equations for mechanisms (i), (iii) and (iv) in table 1 . We also include the equations for the (v) internal friction stress model [9] and (vi) the ideal twinning stress model [10] in table 1. 
Table 1. Predicted and experimental twinning stress for bcc Fe based on several twinning mechanisms. The term $\tau$ represents the critical twinning stress, $\gamma_{\mathrm{sf}}$ represents the stable stacking fault energy, $\gamma_{\mathrm{us}}$ represents the unstable stacking fault energy, $\gamma_{\mathrm{sf}}$ is the stable stacking fault energy, $b$ is the Burgers vector of the twinning fractional, $d$ is the $\left\{\begin{array}{lll}1 & 1 & 2\end{array}\right\}$ interplanar distance in a bcc crystal, $\mu$ is the shear modulus, $\gamma_{\text {Твм }}$ is the twin boundary migration energy and $\tau_{o}$ is an experimental constant.

\begin{tabular}{llll}
\hline Mechanism/model & $\begin{array}{l}\text { Critical twinning stress } \\
\text { expression }\end{array}$ & $\begin{array}{l}\text { Predicted (MPa) } \\
\text { bcc Fe }\end{array}$ & $\begin{array}{l}\text { Experimental (MPa) } \\
\text { bcc Fe }\end{array}$ \\
\hline Pole Mechanism & $=\frac{\gamma_{\mathrm{us}}}{b}$ & 7050 & 170 \\
Dislocation core dissociation & $\tau=\frac{\gamma_{s f}}{3 b}$ & 2500 & 430 \\
Edge dislocation dissociation & $\tau=k_{t} \frac{\mu}{2 \pi(1-v)}$ & 7120 & \\
Internal friction stress & $\tau=\tau_{o}+\frac{1.4 \gamma_{\mathrm{sf}}}{d}$ & 1530 & \\
Theoretical & $\tau$ & $=\frac{\pi \gamma_{\mathrm{TBM}}}{b}$ &
\end{tabular}

The pole mechanism (i) estimates the critical twinning stress, $\tau=\gamma_{\mathrm{us}} / b$, based on the unstable stacking fault energy, which tends to be higher than the experimental value. For example, $\tau=\gamma_{\mathrm{us}} / b$ results in $7050 \mathrm{MPa}\left(\gamma_{\mathrm{us}}=617 \mathrm{~mJ} \mathrm{~m}^{-2}\right.$ for Fe $\left.[11,12]\right)$ while the experimental value for $\mathrm{Fe}$ is $170 \mathrm{MPa}[13,14]$.

The dislocation core dissociation (iii) mechanism listed in table 1 relates the critical twining stress to the stable stacking fault energy, $\gamma_{\mathrm{sf}}\left(\tau=\gamma_{\mathrm{sf}} / 3 b\right)$ Based on this assumption $\left(\gamma_{\mathrm{sf}}=593 \mathrm{~mJ} \mathrm{~m}^{-2}\right.$ for Fe $\left.[11,12]\right)$, the twinning stress for Sleeswyk's three-fold mechanism is estimated to be $2500 \mathrm{MPa}$ for bcc Fe.

The edge dislocation dissociation mechanism (iv) results in an equation of the form $\tau=k_{t} \mu / 2 \pi(1-v)$, where $\mu$ is the shear modulus and $v$ is the Poisson's ratio of the bcc crystal. The mechanism is based on the dissociation of a perfect slip dislocation into three fractional dislocations that rearrange to form a twin, and predicts a twinning stress magnitude of $430 \mathrm{MPa}$. The term $k_{t}$ which appears in this formulation denotes the frictional stress to dislocation glide, and can be fitted only from experiments.

The internal friction stress model (v) considers a twin to nucleate when the applied stress exceeds a critical value $\tau_{o}$ representing the Peierls stress. With the values for $\tau_{o}, \gamma_{\text {sf }}$ and $d$ taken, respectively, as $1400 \mathrm{MPa}, 593 \mathrm{~mJ} \mathrm{~m}^{-2}$ and $1.2 \AA$ in the equation $\tau=\tau_{o}+1.4 \gamma_{\mathrm{sf}} / d$, we obtain a twinning stress of $7120 \mathrm{MPa}$ for bcc Fe. The parameter $d$ in the above equation is the $\left\{\begin{array}{lll}1 & 1 & 2\end{array}\right\}$ interplanar spacing and $\gamma_{\mathrm{sf}}$ is the energy corresponding to the first layer twin stacking fault (to be shown later).

Finally, the theoretical (ideal) twinning stress (vi) can be calculated from the generalized plane fault energy (GPFE) curves with respect to the shear displacement and is in the form $\tau=\pi \gamma_{\mathrm{TBM}} / b$, where $\gamma_{\text {Твм }}$ is the difference between the unstable twin fault energy, $\gamma_{\mathrm{ut}}$, and the stable twin stacking fault energy, $2 \gamma_{\text {tsf }}$. However, in the case of pure Fe, this yields to the theoretical twinning stress of $1530 \mathrm{MPa}$, which is an order of magnitude higher compared to the experimental value.

Overall, the difference between the theoretical twinning stress and the experimental one is substantial, which warrants further investigation into the problem. There are several requirements for a correct estimation of the twinning stress: the first requirement is the consideration of the precise energy path traced by the dislocations leading to the twinning nucleus; the second is the determination of the elastic energy of a multi-layer dislocations 
arrangement. The present model considers the GPFE curve prescribed over the atomic displacements to create the twin nucleus, and the mutual interaction of the fractional dislocations and their elastic energies. We therefore construct an overall energy expression and obtain the twinning stress in bcc metals in excellent agreement with experiments. Since the parameters are derived from atomistic simulations, empirical constants are not necessary.

In addition to the twin stress modeling, we need to precisely determine experimentally the twin nucleation stress. Utilizing novel experimental digital image correlation (DIC) techniques, single-crystal orientations and high-resolution microscopic observations, we validate the modeling efforts with experiments. We make precise measurements of the onset of twinning in $\mathrm{Fe}-50 \% \mathrm{Cr}$ single crystals using DIC for a number of crystal orientations, and establish the critical twinning stress. The experimental observations in conjunction with transmission electron microscopy (TEM) measurements show the activation of a three-fold symmetric slip system conducive to twinning. The dislocation arrangements with twinning employed in the present analysis are discussed in the following sections.

\subsection{Dislocation arrangements in twinning}

Under an applied stress, twinning in bcc metals occurs when three fractional dislocations lying on three $\left\{\begin{array}{lll}1 & 1 & 2\end{array}\right\}$ planes translate along the most stressed $\left\{\begin{array}{lll}1 & 1 & 2\end{array}\right\}$ plane (figure 1$)$. The $\left\langle\begin{array}{llll}1 & 1 & 1\end{array}\right)$ core structure has been studied extensively in bcc crystals [15-20] employing different atomistic methods, and core spreading has been taken into consideration. Of the possible core structures, the [ $\left.\begin{array}{lll}1 & 1 & 1\end{array}\right]$ core of an $(a / 2)\left\langle\begin{array}{llll}1 & 1 & 1\end{array}\right\rangle$ dislocation is considered to dissociate into three $(a / 6)\langle 111\rangle$ fractional dislocations as follows:

$$
\frac{a}{2}\left[\begin{array}{lll}
1 & 1 & 1
\end{array}\right]_{\text {screw }} \rightarrow 3 \times \frac{a}{6}\left[\begin{array}{lll}
1 & 1 & 1
\end{array}\right] .
$$

As Vitek [20] pointed out, unlike the metastable partial dislocations in fcc metals, the fractional dislocations in bcc are associated with an unstable fault energy. The three fractional dislocations possess a configuration symmetric to the $\langle 111\rangle$ screw axis as illustrated in figure $1(a)$. The configuration is stable under zero external stress. However, under an applied stress, the dislocations translate along the most stressed $\left\{\begin{array}{lll}1 & 1 & 2\end{array}\right\}$ plane (in twinning sense) to create a three-layer twin as shown in figures $1(b)$ and $(c)$. Theoretical calculations predict the width $(w)$ of the $(a / 2)\langle 111\rangle$ dissociated core in bcc crystal to be $1 b-2 b$, where $b=((\sqrt{3} a) / 2)$. This leads to the calculation of the separation distance $(x)$ between any pair of fractional dislocations in figure $1(a)$ to be larger than $2 d_{\left\{\begin{array}{ll}1 & 1\end{array}\right\}}$, where $d_{\left\{\begin{array}{ll}1 & 12\end{array}\right\}}$ is the $\left\{\begin{array}{lll}1 & 1 & 2\end{array}\right\}$ interplanar distance. Under the applied stress, dislocations $A$ and $C$ separated by a distance

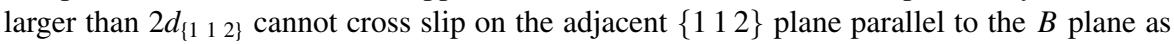
it creates a high-energy fault. Therefore, dislocation $A$ initially glides on its (1 112 ) plane

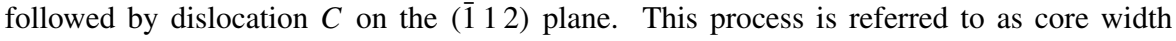
adjustment [7], and is shown by the arrows in figure 1(a). When the two dislocations $A$ and $C$ lie on consecutive $\left\{\begin{array}{lll}1 & 1 & 2\end{array}\right\}$ planes parallel to the $B$ plane as a result of the glide, they eventually cross slip to form a stable three-layer fault (twin). Experimental observations in $\mathrm{Fe}-50 \mathrm{at} \% \mathrm{Cr}$ single crystals in our present study show the activation of the $\left\{\begin{array}{lll}1 & 1 & 2\end{array}\right\}$ slip on the most favorable slip plane followed by other systems. Twin nucleation can occur after three-fold slip systems are present at higher stresses.

\subsection{Twinning energy landscape (GPFE)}

In bcc metals, twins are multi-layer stacking faults created by the passage of $b_{\mathrm{p}}=(a / 6)\langle 111\rangle$ dislocations (' $a$ ' is the bcc lattice constant) on successive $\left\{\begin{array}{lll}1 & 12\end{array}\right\}$ planes [21]. Figure 2 


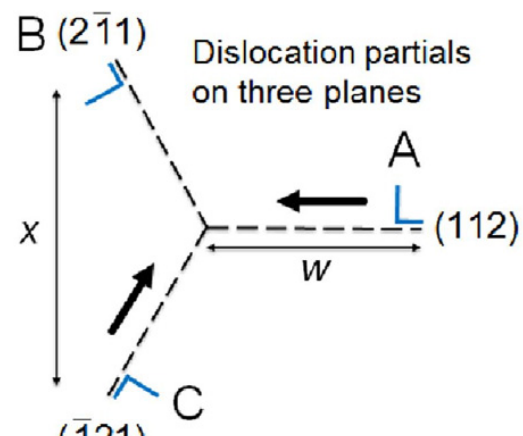

(a)

\section{$\mathrm{C}$ and $\mathrm{A}$ translation to $B$ plane}

B

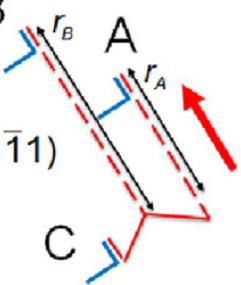

(b)

(c)

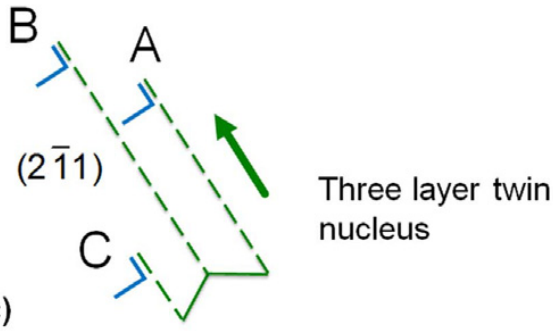

Figure 1. $(a)$ the dissociated $(a / 2)[11 \overline{1}]$ dislocation with fractional dislocations on $\left\{\begin{array}{lll}1 & 1 & 2\end{array}\right\}$ planes represented as $A, B$ and $C(b)$ dislocation configuration on the verge of twinning $\left(r_{\mathrm{c}}=0\right)(c)$ three-layer twin nucleus moving on the $(2 \overline{1} 1)$ plane.

illustrates the sequential process of shearing the consecutive $\left\{\begin{array}{lll}1 & 1 & 2\end{array}\right\}$ planes of a bcc crystal to form a twin. Glide of the first $b_{\mathrm{p}}=(a / 6)\langle 111\rangle$ dislocation on the $\left\{\begin{array}{lll}1 & 1 & 2\end{array}\right\}$ plane creates a onelayer stacking fault, and the second and third dislocations on successive $\left\{\begin{array}{lll}1 & 1 & 2\end{array}\right\}$ planes create two- and three-layer stacking faults, respectively. Further glide of the dislocations (beyond the third layer) on successive $\left\{\begin{array}{lll}1 & 1 & 2\end{array}\right\}$ planes represents twin growth. As shown in figure 2, when the layers $\mathrm{F}-\mathrm{F}$ of a perfect bcc $\left\{\begin{array}{lll}1 & 1 & 2\end{array}\right\}$ stacking sequence are translated through a displacement of $b_{\mathrm{p}}=(a / 6)\langle 111\rangle$ relative to the bottom layers A-E, a one-layer stacking fault is created. Similarly, a successive translation of layers A-F followed by layers B-F creates two-layer and three-layer stacking faults, respectively.

The thinnest, stable multilayer stacking fault on the $\left\{\begin{array}{lll}1 & 1 & 2\end{array}\right\}$ plane is a three-layer fault, and can be considered to be the twin nucleus [22,23]. The increase in additional faults is considered to be twin growth where fault energies remain unaltered during the process. The processes of twin nucleation and growth are distinctly governed by different regions of the GPFE curve [24]. The distinction between these processes is crucial because twin nucleation requires much higher stress than that for growth. It is worth emphasizing that the energy values in figure 3 do not depend on the atomic arrangement for a disordered $\mathrm{Fe}-50$ at $\% \mathrm{Cr}$ alloy (bcc), similar to other bcc alloys considered in the present analysis. This is because MD uses a representative volume consisting of a large number of atoms (in the thousands), and the positions of the substitutional atoms do not affect the energy parameters. 

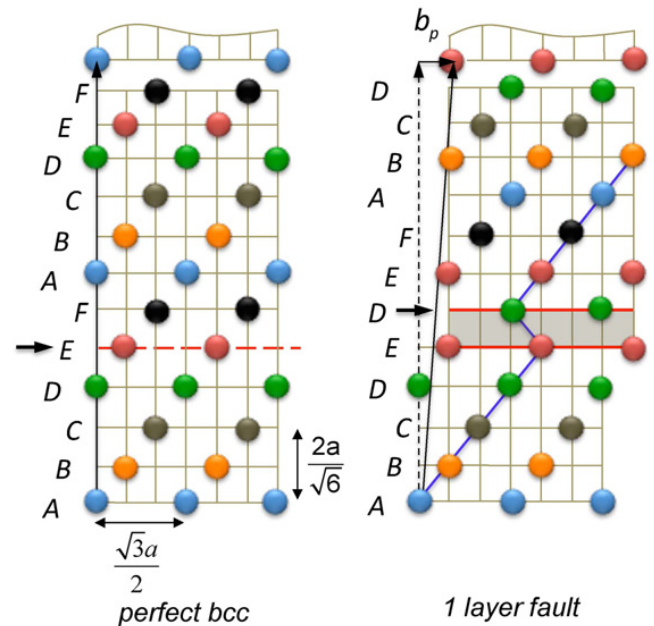

1 layer fault

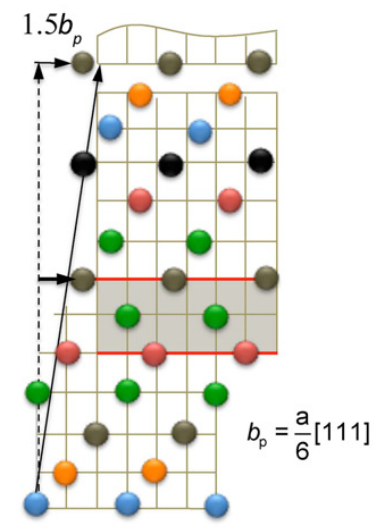

Intermediate 1.5 layer fault
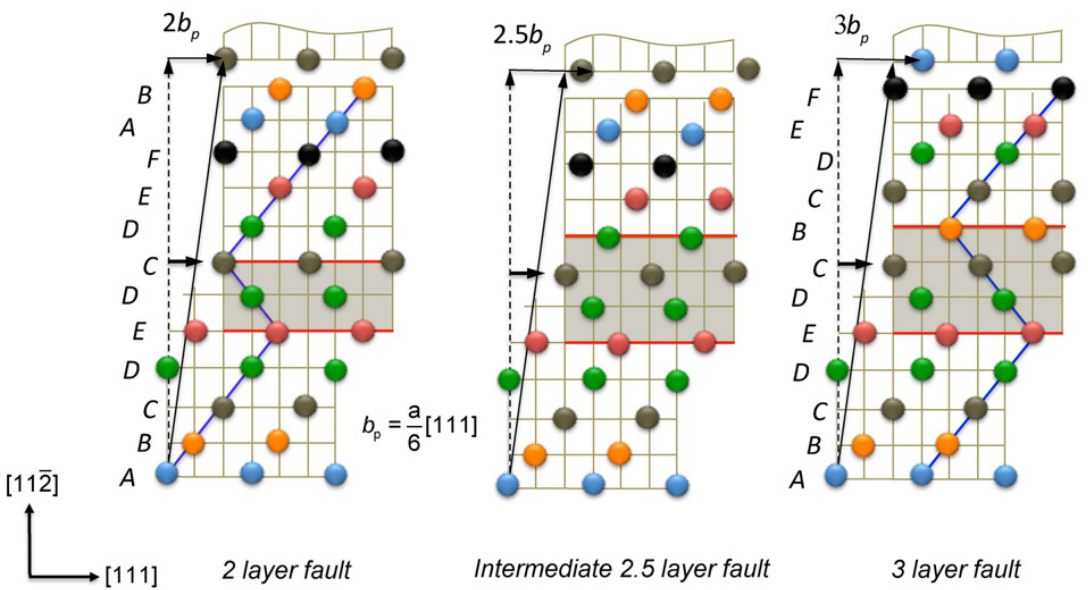

2 layer fault

Intermediate 2.5 layer fault

3 layer fault

Figure 2. $\left\{\begin{array}{llll}1 & 1 & 2\end{array}\right\}\left\langle\begin{array}{llll}1 & 1 & 1\end{array}\right\rangle$ stacking sequence in a bcc crystal. Atoms on different $\left\{\begin{array}{llll}1 & 1 & 2\end{array}\right\}$ planes are represented by different colors. The arrows indicate the passage of the dislocation on successive $\left\{\begin{array}{lll}1 & 1 & 2\end{array}\right\}$ planes creating one-layer, two-layer and three-layer stacking faults, and the intermediate structures (Adapted from [24]).

\section{The current approach}

\subsection{Atomistic simulations}

In the present work, the GPFE curve for $\mathrm{Fe}-50 \mathrm{at} \% \mathrm{Cr}$ was determined with the MD technique employing the embedded atom method (EAM) potential [25]. In order to generate an alloy model for $\mathrm{Fe}-50 \mathrm{at} \% \mathrm{Cr}$, a parallel simulator code was written to create 5040 atoms consisting of two atom types $\left(N_{\mathrm{Fe}}=2520, N_{\mathrm{Cr}}=2520\right)$. The atom types were assigned randomly in the simulation box, but following the bcc crystal coordinates so that the two atomic positions 


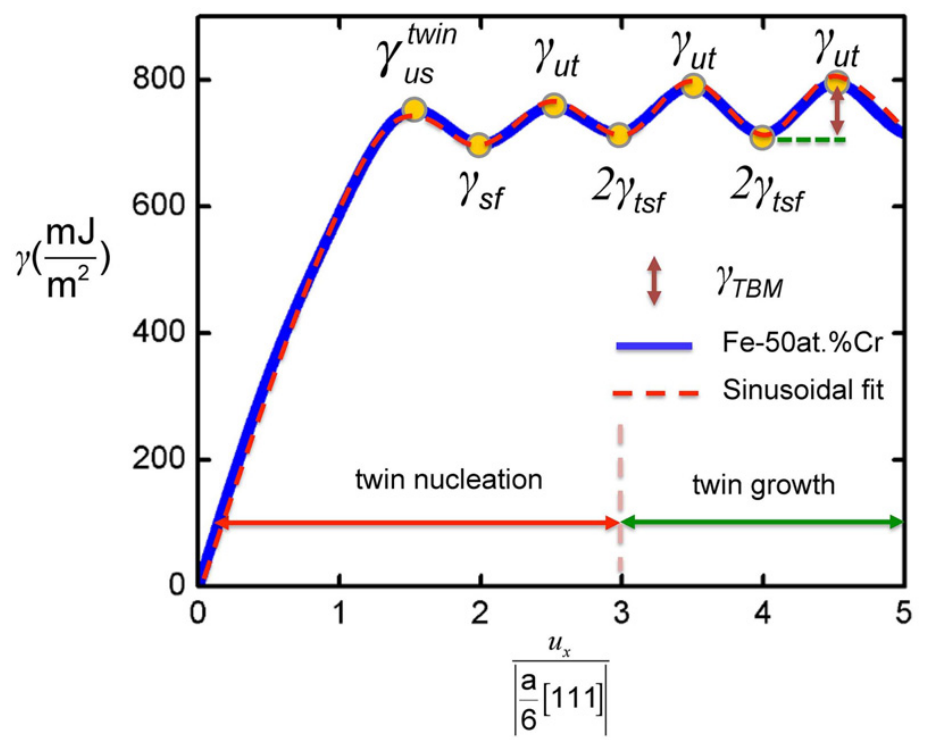

Figure 3. GPFE for $\mathrm{Fe}-50 \% \mathrm{Cr}$ at various normalized shear displacements on successive $\left\{\begin{array}{lll}1 & 1 & 2\end{array}\right\}$ planes calculated in the present study. The normalized displacements and the stacking fault energies corresponding to twin nucleation and twin growth are shown. The dashed lines show the sinusoidal fit to the GPFE response obtained from atomistic calculations.

do not overlap. The box edges were oriented along the [1 111$],\left[\begin{array}{lll}1 & 1 & \overline{2}\end{array}\right]$ and [ $\left[\begin{array}{lll}1 & 1 & 0\end{array}\right]$ directions.

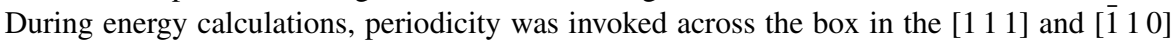
directions. Molecular statics was used to relax the perfect crystal at a temperature of $10 \mathrm{~K}$ to confirm that the crystal was stress free before shearing. To obtain the first layer stacking fault energy value using LAMMPS [26], we first divided the simulation box into upper and lower regions in the $\left\langle\begin{array}{lll}1 & 1 & 2\end{array}\right\rangle$ direction. We then rigidly displaced all the atoms in the upper region with respect to the lower region by a displacement of $b_{p}$. Similarly, to obtain the second-layer fault energy, a new upper region was defined that consisted of only the atoms above but not including the already sheared $\left\{\begin{array}{lll}1 & 1 & 2\end{array}\right\}$ first layer fault. The new region was then displaced by $2 b_{p}$. A similar procedure was repeated to obtain the third, fourth and fifth twin layers. The specifics to carry out these procedures can be found in detail in the LAMMPS manual. We partially minimized the sheared crystal in the $\left\langle\begin{array}{lll}1 & 1 & 2\rangle\end{array}\right\rangle$ direction. This is important to maintain the atoms at their relevant positions, otherwise the atoms move to stable energy positions near the fault during relaxation. If $E_{s}$ is the energy of the deformed lattice corresponding to a shear displacement of $u_{x} / b_{p}$, and $E_{o}$ is that of the perfect lattice, then the stacking fault energy can be calculated as [27]

$$
\gamma=\frac{E_{\mathrm{s}}-E_{\mathrm{o}}}{A_{\{112\}}}
$$

where $A_{\{112\}}$ is the area of the $\left\{\begin{array}{lll}1 & 12\end{array}\right\}$ plane of the simulation box.

It is important to note that 5040 atoms were large enough to obtain the converged GPFE values. This was verified with independent simulations with number of atoms ranging from 400 to 2776586 . 
The GPFE curves for other bcc alloys were obtained in a similar manner employing various potentials [28-30]. The potentials used in the present calculations yield physical properties [25, 28-31] (such as elastic constants, lattice parameters, cohesive energy, solid solution lattice parameters, etc), in reasonable agreement with the experimental data [32,33] or other empirical methods and DFT calculations [34,35].

Figure 3 illustrates GPFE for the Fe-50 at $\% \mathrm{Cr}$ alloy where $\gamma_{\text {usf }}^{t \text { win }}$ is defined as the unstable stacking fault energy and represents the energy barrier that the $(a / 6)\langle 111\rangle$ dislocation must overcome to create the first-layer stacking fault. Similarly, $\gamma_{\mathrm{sf}}$ is the stable stacking fault energy, $\gamma_{\mathrm{ut}}$ is the unstable twinning fault energy, $\gamma_{\mathrm{TBM}}$ is the twin migration energy and $2 \gamma_{\text {tsf }}$ is the stable twin stacking fault energy. It has been observed in our calculation that $\gamma_{\mathrm{ut}}$ does not change beyond the third layer. Figure 3 also demonstrates that the sinusoidal series fit (shown by the dashed curves) can accurately capture the GPFE response. The fitting equations will be discussed later.

\subsection{Experimental procedures and observations}

Single crystals of $\mathrm{Fe}-50 \mathrm{at} \% \mathrm{Cr}$ with loading axes parallel to the [1 $\overline{1} 1],\left[\begin{array}{lll}1 & 0 & 1\end{array}\right]$ and $\left[\begin{array}{ll}0 & 0\end{array}\right]$ orientations were used to study the evolution of twinning. The Bridgeman technique was employed to manufacture the samples in an He atmosphere. The samples of dimensions $4 \mathrm{~mm} \times 4 \mathrm{~mm} \times 10 \mathrm{~mm}$ were cut using electro-discharge machining (EDM). Electron back scattering diffraction (EBSD) was used to determine the normal and transverse orientations of the samples. The samples were solution annealed at $900^{\circ} \mathrm{C}$ for $1 \mathrm{~h}$, and water quenched prior to loading. Displacement and strain-controlled experiments were conducted for compressive and tensile tests, respectively, using a servo hydraulic load frame at room temperature at a strain rate of $5 \times 10^{-3} \mathrm{~s}^{-1}$. The DIC technique, combined with scanning electron microscopy (SEM), was used to study the nucleation of slip and the evolution of twinning at different points of the loading curve as illustrated in figure 4 . Ex situ high-resolution images $(0.44 \mu \mathrm{m} / \mathrm{pixel})$ were obtained utilizing an optical microscope. The high-resolution DIC strain measurements elucidated the onset of slip, twin nucleation and twin growth at different points of the loading curve. For higher-resolution microstructural studies, TEM was employed. For TEM work, $1 \mathrm{~mm}$ thick slices were cut parallel to the loading direction of the crystal and then mechanically ground and polished down to a foil thickness of $100 \mathrm{~mm}$. Large electron-transparent areas were obtained by conventional twin-jet electropolishing with an electrolyte consisting of 5 pct perchloric acid in methanol. Electropolishing was done at a temperature of $-23^{\circ} \mathrm{C}$ and a potential of $21 \mathrm{~V}$. Whenever possible, two-beam imaging conditions were employed for imaging. The burgers vectors were determined using the $g \cdot b=0$ invisibility criteria. Also, the line directions of the dislocations were determined by tilting to different orientations. In the current paper, we present the extensive TEM and DIC results only for [ $\left[\begin{array}{lll}1 & 1 & 1\end{array}\right]$ compressive loading to identify the activated twinning systems. The experimentally observed twin systems listed in table 2 for [ $\left[\begin{array}{lll}0 & 1 & 0\end{array}\right]$ and [ $\left[\begin{array}{lll}1 & 0 & 1\end{array}\right]$ orientations were identified using a similar procedure.

Figure 4 shows the stress-strain response of $\mathrm{Fe}-50$ at.\% $\mathrm{Cr}$ single crystal subjected to compression in [1 $1 \overline{1} 1]$ orientation. High-resolution DIC measurements reveal slip on the $\left[\begin{array}{lll}\overline{1} & 1 & 1\end{array}\right]\left(\begin{array}{lll}2 & \overline{1} & 1\end{array}\right)$ system $($ Schmid Factor $(\mathrm{SF})=0.30)$ at a critical resolved shear stress $\left(\mathrm{CRSS}, \tau^{\mathrm{s}}\right)$ of $88 \mathrm{MPa}$ (inset marked A). The twin develops on the same system when the strain reaches $1.1 \%$ and is characterized by a sudden drop in the load. The nucleation of the twin occurred at a $\operatorname{CRSS}\left(\tau^{\mathrm{T}}\right)$ of $202 \mathrm{MPa}$.

An examination of the TEM images of figures $5(a)$ and $(b)$ and the optical image of figure 6 for the case of [ $\left.\begin{array}{lll}1 & \overline{1} & 1\end{array}\right]$ compressive loading orientation shows evidence of the activated $\left\{\begin{array}{lll}1 & 1 & 2\end{array}\right\}$ slip systems (shown by dashed colored lines) prior to twinning. It is important to note that 


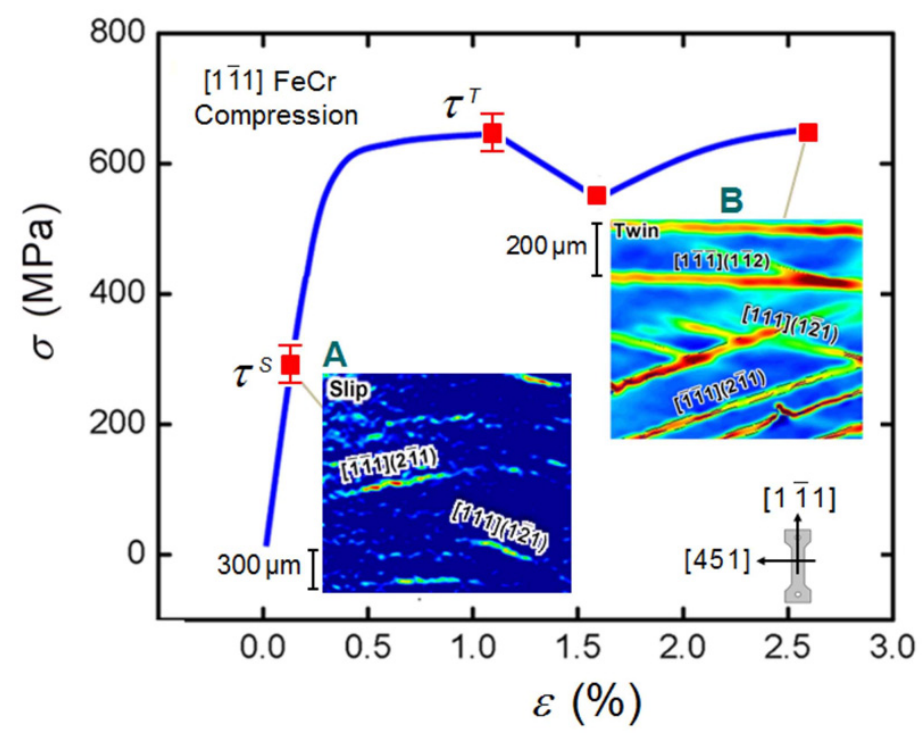

Figure 4. Stress-strain curve for a $\mathrm{Fe}-50$ at.\% Cr single crystal under [ $\left[\begin{array}{lll}1 & 1 & 1\end{array}\right]$ compressive loading corresponding to six different tests. The DIC images marked A and B show the nucleation of $\left(\begin{array}{lll}2 & \overline{1} & 1\end{array}\right)\left[\begin{array}{lll}\overline{1} & \overline{1} & 1\end{array}\right]$ and $\left(\begin{array}{lll}1 & \overline{2} & 1\end{array}\right)\left[\begin{array}{lll}1 & 1 & 1\end{array}\right](\mathrm{SF}=0.30)$ slip and the onset of twinning on the same systems, respectively.

Table 2. Experimentally observed slip systems and their corresponding SF for a $\mathrm{Fe}-50$ at.\% Cr single crystal subjected to tensile and compressive loading in different orientations. The activated slip systems possess symmetric configuration which is consistent with the bcc twinning mechanism proposed in this work.

\begin{tabular}{|c|c|c|c|c|c|}
\hline & Loading orientation & Activated systems & SF & Observed twin & $\tau^{\mathrm{T}}(\mathrm{MPA})$ \\
\hline \multirow[t]{6}{*}{ Compression } & {$\left[\begin{array}{lll}1 & 0 & 1\end{array}\right]$} & {$\left[\begin{array}{lll}1 & 1 & 1\end{array}\right]\left(\begin{array}{lll}\overline{2} & 1 & 1\end{array}\right)$} & 0.24 & {$\left[\begin{array}{lll}1 & 1 & 1\end{array}\right]\left(\begin{array}{lll}1 & \overline{2} & 1\end{array}\right)$} & $194 \pm 8$ \\
\hline & & {$\left[\begin{array}{llll}1 & 1 & 1\end{array}\right]\left(\begin{array}{lll}1 & \overline{2} & 1\end{array}\right)$} & 0.47 & & \\
\hline & & {$\left[\begin{array}{llll}1 & 1 & 1\end{array}\right]\left(\begin{array}{lll}1 & 1 & \overline{2}\end{array}\right)$} & 0.24 & & \\
\hline & {$\left[\begin{array}{lll}1 & \overline{1} & 1\end{array}\right]$} & {$\left[\begin{array}{llll}\overline{1} & \overline{1} & 1\end{array}\right]\left(\begin{array}{lll}2 & \overline{1} & 1\end{array}\right)$} & 0.30 & {$\left[\begin{array}{llll}\overline{1} & \overline{1} & 1\end{array}\right](2 \overline{1} 1)$} & $203 \pm 13$ \\
\hline & & {$\left[\begin{array}{llll}\overline{1} & \overline{1} & 1\end{array}\right]\left(\begin{array}{lll}1 & 2 & 1\end{array}\right)$} & 0.15 & & \\
\hline & & {$\left[\begin{array}{llll}\overline{1} & \overline{1} & 1\end{array}\right]\left(\begin{array}{lll}1 & 1 & 2\end{array}\right)$} & 0.15 & & \\
\hline \multirow[t]{6}{*}{ Tension } & {$\left[\begin{array}{lll}0 & \overline{1} & 0\end{array}\right]$} & {$\left[\begin{array}{lll}1 & \overline{1} & 1\end{array}\right]\left(\begin{array}{lll}2 & 1 & \overline{1}\end{array}\right)$} & 0.24 & {$\left[\begin{array}{llll}1 & \overline{1} & 1\end{array}\right]\left(\begin{array}{lll}1 & 2 & 1\end{array}\right)$} & $177 \pm 13$ \\
\hline & & {$\left[\begin{array}{llll}1 & \overline{1} & 1\end{array}\right]\left(\begin{array}{lll}1 & 2 & 1\end{array}\right)$} & 0.47 & & \\
\hline & & {$\left[\begin{array}{llll}1 & \overline{1} & 1\end{array}\right]\left(\begin{array}{lll}\overline{1} & 12\end{array}\right)$} & 0.24 & & \\
\hline & & {$\left[\begin{array}{lll}\overline{1} & 1 & 1\end{array}\right]\left(\begin{array}{lll}1 & \overline{1} & 2\end{array}\right)$} & 0.24 & & \\
\hline & & {$\left[\begin{array}{lll}\overline{1} & 1 & 1\end{array}\right]\left(\begin{array}{llll}2 & 1 & 1\end{array}\right)$} & 0.24 & & \\
\hline & & & 0.47 & & \\
\hline
\end{tabular}

the TEM images in figure 5 are essentially meant to elucidate the three symmetric $\left\{\begin{array}{lll}1 & 1 & 2\end{array}\right\}$ slip planes activated under different loading orientations (consistent with figure 1 $(a)$ ), and not to demonstrate the entire twinning mechanism. It is worth emphasizing that other slip/twin systems are also activated under these loading orientations; nevertheless, we emphasize only 


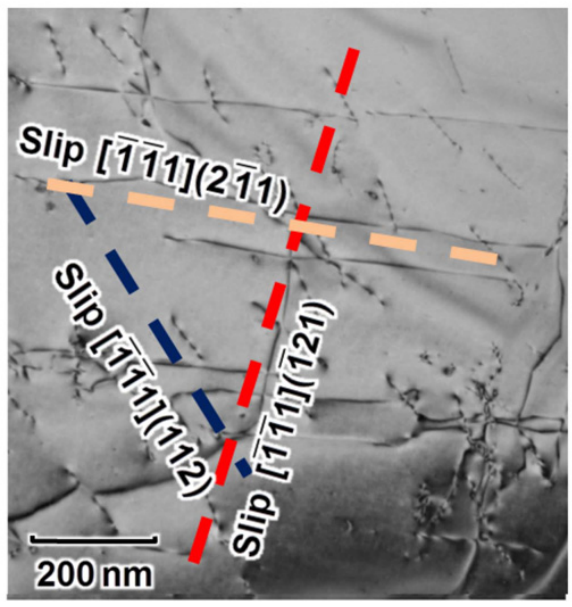

(a)

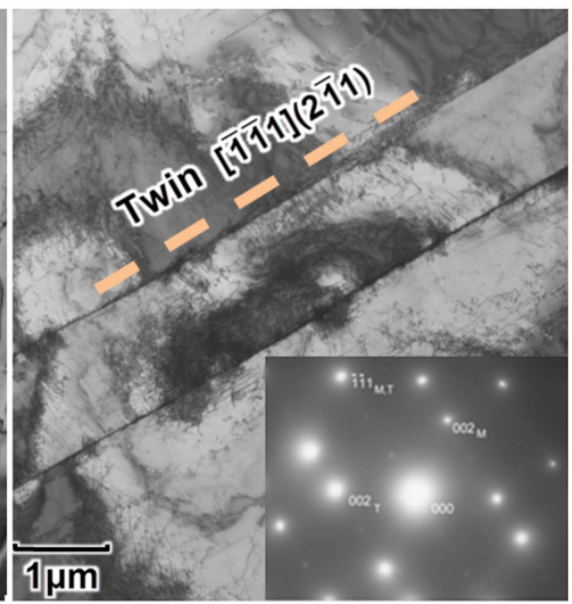

(b)

Figure 5. (a) TEM image of a $\mathrm{Fe}-50 \% \mathrm{Cr}$ single crystal illustrating the activated $\left\{\begin{array}{llll}1 & 1 & 2\end{array}\right\}\left\langle\begin{array}{llll}1 & 1 & 1\end{array}\right\rangle$ slip systems (shown by dashed colored lines) under [1 $\left.1 \overline{1} 1\right]$ compressive loading. The image was recorded under multi-beam conditions to bring the dislocations from different systems into contrast. (b) Twinning is observed on the $\left(\begin{array}{lll}2 & 1 & 1\end{array}\right)\left[\begin{array}{lll}\overline{1} & \overline{1} & 1\end{array}\right]$ system corresponding to the highest SF of 0.30 for the same loading orientation. The inset shows the corresponding diffraction pattern (lower right image), where the subscripts $\mathrm{M}$ and $\mathrm{T}$ denote matrix and twin, respectively. The electron beam is parallel to $\left[\begin{array}{lll}1 & 1 & 0\end{array}\right]_{M}$.

those systems belonging to a distinct three-fold symmetric configuration. Twinning is observed on the $[\overline{1} \overline{1} 1](2 \overline{1} 1)(\mathrm{SF}=0.30)$ system, which corresponds to the maximum resolved

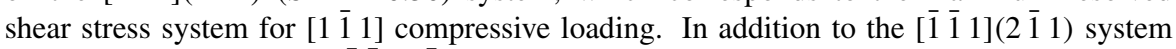
$(\mathrm{SF}=0.30)$, the low SF $\left[\begin{array}{lll}1 & 1 & 1\end{array}\right]\left(\begin{array}{ll}1 & 2 \\ 1\end{array}\right)(\mathrm{SF}=0.15)$ slip system is also observed to activate during loading.

\section{Modeling of the twin nucleation stress}

We analyze the elastic interactions of the dislocations forming the twin nucleus at a mesoscale and incorporate the GPFE obtained at an atomistic level (MD) to predict the twinning stress. We therefore employ the following findings in our analysis corresponding to two different length scales: (i) $(a / 6)\langle 111\rangle$ fractional dislocations in a bcc lie on the three symmetric $\left\{\begin{array}{lll}1 & 1 & 2\end{array}\right\}$ planes as shown in figure $1(a)$, (ii) the $(a / 6)\langle 111\rangle$ fractional dislocations under external stress gradually reorient themselves along the most stressed $\left\{\begin{array}{lll}1 & 1 & 2\end{array}\right\}$ plane to attain a stable three-layer stacking fault (twin) as shown by figures $1(b)$ and $(c)$ and (iii) the $(a / 6)\langle 111\rangle$ dislocations have to overcome an energy barrier to nucleate a twin that is quantitatively represented by the GPFE. We then develop an analytical expression to determine the twin nucleation stress in bcc metals. We do so by differentiating the total energy of the dislocations of the twin nucleus with respect to their positions $r_{A}$ and $r_{B}$ (see figure $1(c)$ ).

Figure $1(b)$ depicts the arrangement of the $(a / 6)\langle 111\rangle$ dislocations on the verge of twinning. The total energy of the configuration in figure $1(b)$ can be expressed as the summation 


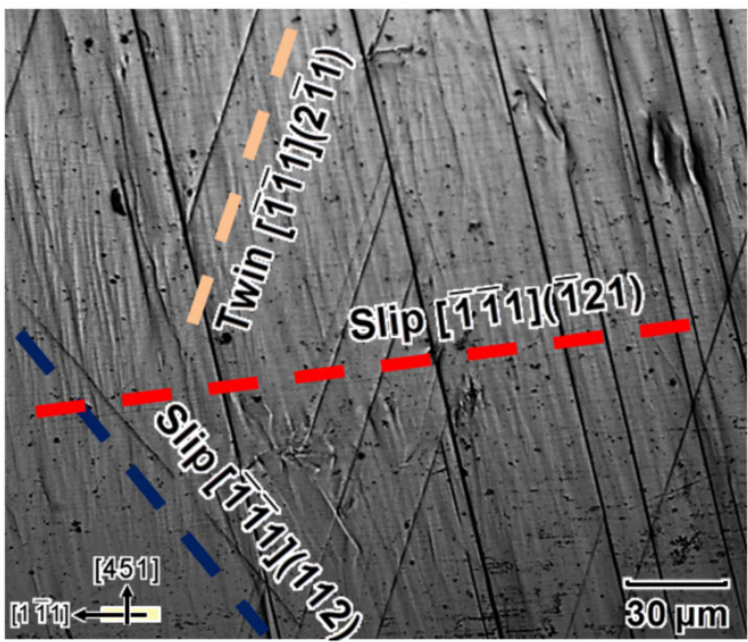

Figure 6. Micrograph of a $\mathrm{Fe}-50$ at.\% $\mathrm{Cr}$ single crystal illustrating the activated $\left\{\begin{array}{llll}1 & 1 & 2\end{array}\right\}\left\langle\begin{array}{llll}1 & 1 & 1\end{array}\right\rangle$ slip systems possessing a three-fold symmetry configuration (shown by the dashed colored lines) under [ $\left.\begin{array}{lll}1 & 1 & 1\end{array}\right]$ compressive loading. Twinning is observed on

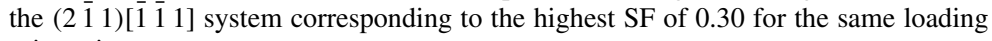
orientation.

of (i) the energy due to elastic interactions of the dislocations forming the twin nucleus, (ii) the line energies of all dislocations and (iii) the twinning energy landscape traversed by the dislocations represented by the area under the GPFE curve (iv) minus the work done by the resolved shear stress. The total energy can be expressed as

$$
E_{\text {total }}=E_{\text {interaction }}+E_{\text {line }}-W_{\mathrm{T}}+E_{\mathrm{GPFE}} .
$$

If we consider the interaction of every $(a / 6)\langle 111\rangle$ dislocation with every other dislocation forming the twin nucleus, then the interaction energy of the configuration in figure $1(b)$ can be expressed as

$$
E_{\text {interaction }}=-\frac{G_{\{112\}\langle 111\rangle} b_{\mathrm{p}}^{2}}{2 \pi}\left(\ln \left[\frac{r_{B}-r_{A}}{r_{o}}\right]+\ln \left[\frac{r_{B}}{2 r_{o}}\right]+\ln \left[\frac{r_{A}}{r_{o}}\right]\right),
$$

where $G_{\{112\}\langle 111\rangle}$ is the shear modulus on the activated $\left\{\begin{array}{lll}1 & 1 & 2\end{array}\right\}$ plane, $b_{p}$ is the burgers vector of the twinning dislocation, $r_{A}$ and $r_{B}$ are the equilibrium positions of the dislocations under applied stress and $r_{o}$ is the equilibrium position under zero external stress. Since the potentials in the present study predict the elastic constants in good agreement with the experimental values, we utilized them further to calculate the shear moduli $G_{\{112\}\langle 111\rangle}$ of $\mathrm{Fe}, \mathrm{Fe}-50 \mathrm{at} \% \mathrm{Cr}$, $\mathrm{Fe}-25 \mathrm{at} \% \mathrm{Ni}$ and $\mathrm{Fe}-3 \mathrm{at} \% \mathrm{~V}$ as $40 \mathrm{GPa}, 61 \mathrm{GPa}, 31 \mathrm{GPa}$ and $57 \mathrm{GPa}$, respectively.

The energy contribution due to the GPFE curve in figure 3 can be divided into two parts: (i) the energy required to create a stable stacking fault, $\gamma_{\mathrm{sf}}$, and (ii) the energy required to nucleate a twin. The energy due to the GPFE curve can expressed as

$$
E_{\mathrm{GPFE}}=E_{\mathrm{sf}}+E_{\mathrm{twin}} .
$$

Equation (3) reveals that different regions of the GPFE curve are associated with slip and twinning processes. The rationale behind the decomposition is that slip and twinning are 
competitive mechanisms [3,24], and $E_{\mathrm{sf}}$ does not contribute to twin nucleation. In order to elucidate their role quantitatively, we express $\gamma_{\mathrm{GPFE}}$ using sine analytical functions as follows:

$$
\begin{aligned}
& \gamma_{\mathrm{sf}}(\lambda(x))= \gamma_{\mathrm{us}}^{\mathrm{twin}} \sin [0.3 \pi \lambda] \quad \text { for } 0 \leqslant \lambda<1.5 \quad \lambda(x)=\frac{u_{x}}{b_{\mathrm{p}}}, \\
&\left.\gamma_{\mathrm{sf}}(\lambda(x))=\frac{1}{2}\left(\gamma_{\mathrm{us}}^{\mathrm{twin}}+\gamma_{\mathrm{sf}}\right)+\left(\gamma_{\mathrm{us}}^{\mathrm{twin}}-\frac{\gamma_{\mathrm{us}}^{\mathrm{twin}}+\gamma_{\mathrm{sf}}}{2}\right) \sin [2 \pi(\lambda(x))-1.25]\right) \quad \text { for } \\
& 1.5 \leqslant \lambda(x)<2, \\
& \gamma_{\mathrm{twin}}(\lambda(x))=\left.\frac{1}{2}\left(\gamma_{\mathrm{ut}}+\gamma_{\mathrm{sf}}\right)+\left(\gamma_{\mathrm{ut}}-\frac{\gamma_{\mathrm{ut}}+\gamma_{\mathrm{sf}}}{2}\right) \sin [2 \pi(\lambda(x)-1.21)]\right) \quad \text { for } 2 \leqslant \lambda(x)<2.5, \\
& \gamma_{\mathrm{twin}}(\lambda(x))=\left.\frac{1}{2}\left(\gamma_{\mathrm{ut}}+2 \gamma_{\mathrm{sf}}\right)+\frac{1}{2}\left(\gamma_{\mathrm{ut}}-2 \gamma_{\mathrm{tsf}}\right) \sin [2 \pi(\lambda(x)-1.22)]\right) \quad \text { for } 2.5 \leqslant \lambda(x)<N .
\end{aligned}
$$

In the above equations, $\lambda$ is the normalized shear displacement of the two halves of the crystal due to the passage of the dislocation $b_{\mathrm{p}}=a / 6\langle 111\rangle$. We express the energy contributions $E_{\mathrm{sf}}$ and $E_{\mathrm{twin}}$ of the GPFE curve as follows:

$$
\begin{aligned}
& E_{\mathrm{sf}}=\int_{0}^{r_{A}} \gamma_{\mathrm{sf}} \mathrm{d} x, \\
& E_{\mathrm{twin}}=\int_{0}^{r_{A}} \gamma_{\mathrm{GPFE}} \mathrm{d} x-\int_{0}^{r_{A}} \gamma_{\mathrm{sf}} \mathrm{d} x .
\end{aligned}
$$

In the above expressions, $E_{\mathrm{sf}}$ is the area under the $\gamma_{\mathrm{sf}}$ curve and represents the energy required by the $(a / 6)\langle 111\rangle$ dislocation to overcome the unstable stacking fault energy barrier $\gamma_{\mathrm{ut}}$ to create a stable stacking fault. Similarly, $E_{\text {twin }}$ is the area under the $\gamma_{\text {twin }}$ curve and represents the energy required by the $(a / 6)\langle 111\rangle$ dislocation to overcome the twin nucleation barrier $\gamma_{\mathrm{ut}}$ in order to create multi-layer stable stacking faults (twin). The energy contribution due to the twin nucleation barrier $\gamma_{\mathrm{ut}}$ can be interpreted as the sum of the twin boundary formation energy and twin boundary migration energy as follows [10]:

$$
\gamma_{\mathrm{ut}}=2 \gamma_{\mathrm{tsf}}+\gamma_{\mathrm{TBM}}
$$

The justification behind the above expression is that in order to extend the boundary of a stable stacking fault by one layer, the $(a / 6)\left\langle\begin{array}{lll}1 & 1 & 1\rangle\end{array}\right)$ dislocation has to overcome the energy barrier represented by $\gamma_{\text {TвM }}$. The newly formed layer will have a stacking fault energy equal to $2 \gamma_{\text {tsf }}$. This implies that once a twin is nucleated, the stable energy corresponding to the three-layer fault (twin) is $2 \gamma_{\text {tsf }}$. This stable energy value $2 \gamma_{\text {tsf }}$ does not change when new faults are created by the passage of additional fractional dislocations on the parallel $\left\{\begin{array}{lll}1 & 1 & 2\end{array}\right\}$ planes consecutive to an already existing fault.

The applied resolved shear stress $\tau_{\mathrm{RSS}}$ helps to overcome the twinning energy barrier. The work done in moving dislocation $A$ from its equilibrium position $r_{o}$ to the final position $r_{A}$ and dislocation $B$ from $2 r_{o}$ to $r_{B}$ on the verge of twinning $\left(r_{\mathrm{c}}=0\right)$ in figure 7 is given by

$$
W_{\tau}=\frac{1}{2} \tau_{\mathrm{RSS}} b\left(r_{A}-r_{o}\right) \sin [2 \alpha]-\frac{1}{2} \tau_{\mathrm{RSS}} b\left(r_{B}-2 r_{o}\right) \sin [2 \alpha],
$$

where $\alpha$ is the angle between the stress axis and normal to the twin plane.

The interaction energy between dislocations as a function of the separation distance $(R)$ can be expressed as the increase in the energy of the system when the dislocations are brought together from some arbitrary value $R_{a}$ to a final separation distance $R$ [36]. At zero external stress, the dislocations $A, B$ and $C$ in figure 7 are initially separated by a distance $r_{o}$ [6]. Under external stress, the separation distance between fractional dislocations $A$ and $B$ is $r_{B}-r_{A}$, and 


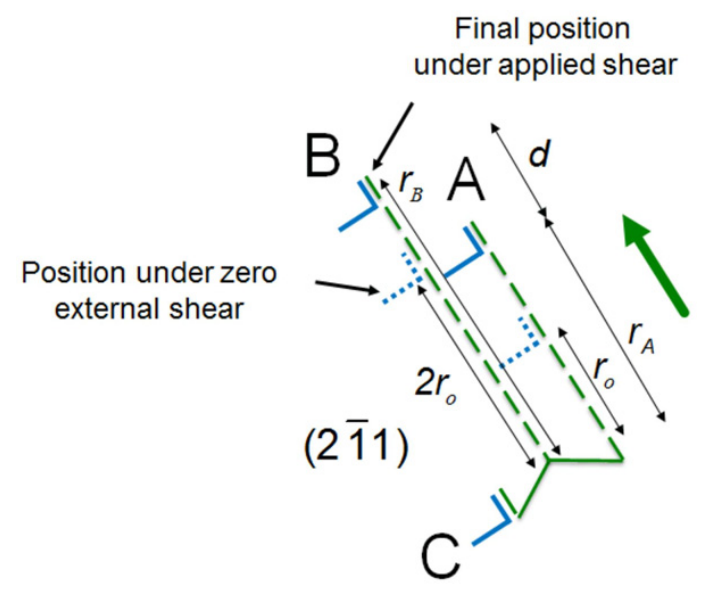

Figure 7. Positions of the dislocations $A$ and $B$ under zero external shear stress and with an applied shear stress. The separation distance between the dislocations $A$ and $B$ is $d$ on the verge of twinning $\left(r_{\mathrm{c}}=0\right)$.

that of $A$ and $C$ is $r_{A}$ with the partial dislocation $C$ being located at $r_{\mathrm{c}}=0$. Following Hirth and Lothe [36], the total energy of the partial dislocations in figure 7 can be written as

$$
\begin{aligned}
E_{\text {total }}=-\frac{G b^{2}}{2 \pi} & \left(\ln \left[\frac{r_{B}-r_{A}}{r_{o}}\right]+\ln \left[\frac{r_{B}}{r_{o}}\right]+\ln \left[\frac{r_{A}}{r_{o}}\right]\right)+n \alpha G b^{2}-\tau_{\mathrm{RSS}} b\left(r_{A}-r_{o}\right) \\
& -\tau_{\mathrm{RSS}} b\left(r_{B}-2 r_{o}\right)+\left(\int_{0}^{r_{A}} \gamma_{\mathrm{GPFE}} \mathrm{d} x-\int_{0}^{r_{A}} \gamma_{s f} \mathrm{~d} x\right),
\end{aligned}
$$

where $n \alpha G b_{\mathrm{p}}^{2}$ is the total line energy of the $n$ dislocations ( $n=3$ in the present analysis). The value of $\alpha$ is taken to be 0.5 [37]. The details of each of the energy terms in equation (8) can be found elsewhere [27,38-41]. We minimized the total energy expression by differentiating it with respect to $r_{A}$ and $r_{B}$ (refer to appendix A1 for the calculations) to numerically solve for the positions of dislocations $A$ and $B$ on the verge of twinning. Having determined positions $r_{A}$ and $r_{B}$, we calculated the separation distance $d$ between the dislocations and derived an analytical expression for critical twin nucleation stress $\tau_{\text {critical }}$ as follows:

$$
\tau_{\text {critical }}=\frac{1}{b}\left\{\gamma_{\mathrm{twin}}^{\prime}-\frac{G b^{2}}{2 \pi}\left(\frac{3-2 \sqrt{3}}{(\sqrt{3}-1) d}\right)\right\} .
$$

$\gamma_{\text {twin }}^{\prime}$ in equation (9) is obtained by the differentiation of $E_{\text {twin }}$ in equation (5) with respect to the position $r_{A}$ and can be written as

$\gamma_{\mathrm{twin}}^{\prime}=\left(\gamma_{\mathrm{ut}}-\frac{\gamma_{\mathrm{ut}}+\gamma_{\mathrm{sf}}}{2}\right) \sin 2 \pi[2.5-1.21]+\frac{1}{4}\left(2 \gamma_{\mathrm{ut}}-2 \gamma_{\mathrm{tsf}}\right) \sin 2 \pi[N-1.22]$,

where $N$ corresponds to the number of layers of the twin nucleus $(N=3$ in the present analysis). Expressions (9) and (10) reveal that the critical stress required for twinning in bcc metals depends on the fault energies $\gamma_{\mathrm{sf}}, \gamma_{\mathrm{ut}}, 2 \gamma_{\mathrm{tsf}}$, the shear modulus $G_{\{112\}\langle 111\rangle}$ and the distance $d$ between dislocations $A$ and $B$ on the verge of twinning, i.e. $\tau_{\text {crtical }}=$ $\tau\left(\gamma_{\mathrm{sf}}, \gamma_{\mathrm{ut}}, 2 \gamma_{\mathrm{sf}}, G, d\right)$. These parameters can be obtained by means of atomistic calculations, thus eliminating the need for any empirical constants in our analysis. It is important to note that the twinning stress expression (9) does not incorporate the strain rate dependence. 


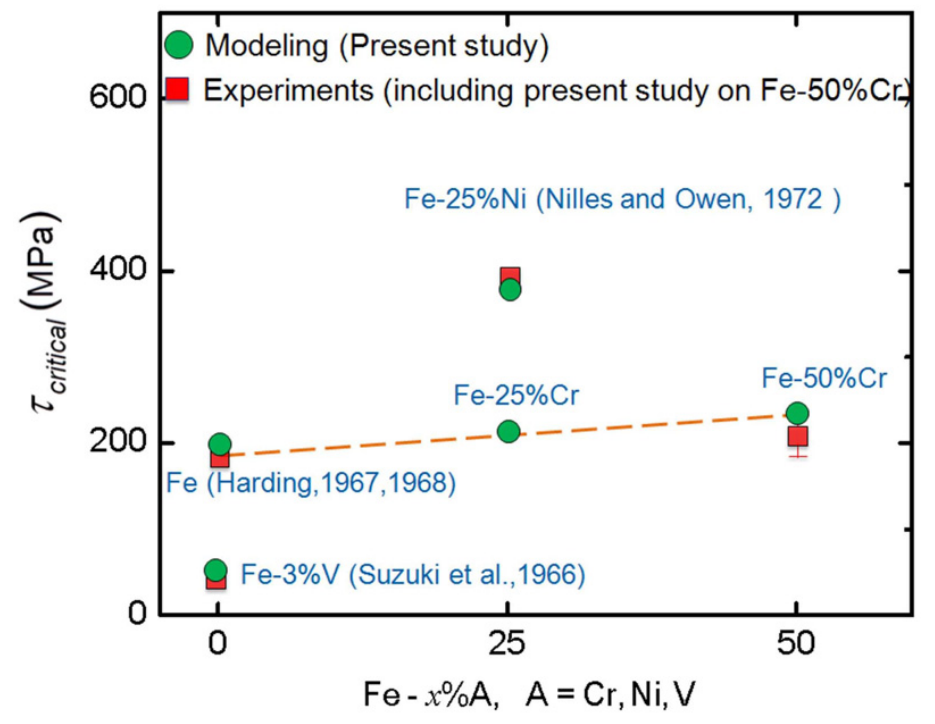

Figure 8. Predicted and experimental $[9,30-32,38]$ (including present results on $\mathrm{Fe}-50$ at.\% $\mathrm{Cr}$ ) twinning stresses for $\mathrm{Fe}$ and Fe-based bcc alloys. The results for $\mathrm{Fe}-50$ at. $\% \mathrm{Cr}$ is an average of 19 tests. The magnitude of the twinning stresses predicted using our analysis is in close agreement to the experimentally measured values. Dashed lines are shown to aid the eye.

\section{Results and discussion}

Figure 8 represents the predicted and experimental $[3,9,13,14,42]$ twinning stresses for a number of Fe-based bcc alloys. We compare our results with the experimental data showing excellent agreement. We observe the trend of the magnitude of twinning stresses for $\mathrm{FeCr}$ alloys using our analysis and experiments, and we conclude that an increase in the chromium content increases the twinning stress. The increase in twinning stress can be attributed to an elevated $\gamma_{\text {TBM }}$ for alloys with relatively higher chromium content. In particular, the present modeling explicitly illustrates the dependence of the twinning stress on the important GPFE parameters $\left(\gamma_{\mathrm{sf}}, \gamma_{\mathrm{ut}}, 2 \gamma_{\mathrm{tsf}}\right)$, which, in fact, could not be captured by other twinning mechanisms, including Sleeswyk [6]. Evidently, figure 9 shows that the nucleation stresses are higher for alloys with higher $\gamma_{\mathrm{TBM}}$ to first approximation; however, the critical twinning stress depends on several other parameters as well, represented by equations (9) and (10). Table 3 shows the predicted stacking fault energies for $\mathrm{Fe}$ and Fe-based alloys where the fault energies are higher for $\mathrm{Fe}-50 \mathrm{at} \% \mathrm{Cr}$ compared to $\mathrm{Fe}$ obtained through the same $\mathrm{FeCr}$ potential. In the present work, we mentioned that $\gamma_{\mathrm{ut}}$ is the sum of the twin boundary formation energy $2 \gamma_{\text {tsf }}$ and twin boundary migration energy $\gamma_{\mathrm{TBM}}$. In order to create a new layer of the stacking fault, the $(a / 6)\left\langle\begin{array}{lll}1 & 1 & 1\end{array}\right\rangle$ dislocation has to overcome the energy barrier represented by $\gamma_{\text {TBM. }}$. With an increase in $\gamma_{\mathrm{TBM}}$, the stress required to create the additional fault increases. Therefore, the higher twin nucleation stress in $\mathrm{Fe}-50 \mathrm{at} \% \mathrm{Cr}$ compared to pure $\mathrm{Fe}$ can be attributed to the increased $\gamma_{\mathrm{TBM}}$ of $\mathrm{Fe}-50 \mathrm{at} \% \mathrm{Cr}$. The increase in $\gamma_{\mathrm{TBM}}$ may be the result of a local distortion in the arrangement of atoms when substitutional $\mathrm{Cr}$ atoms are introduced in the crystal [35]. Atomistic simulations and experimental observations have also been shown to increase the 


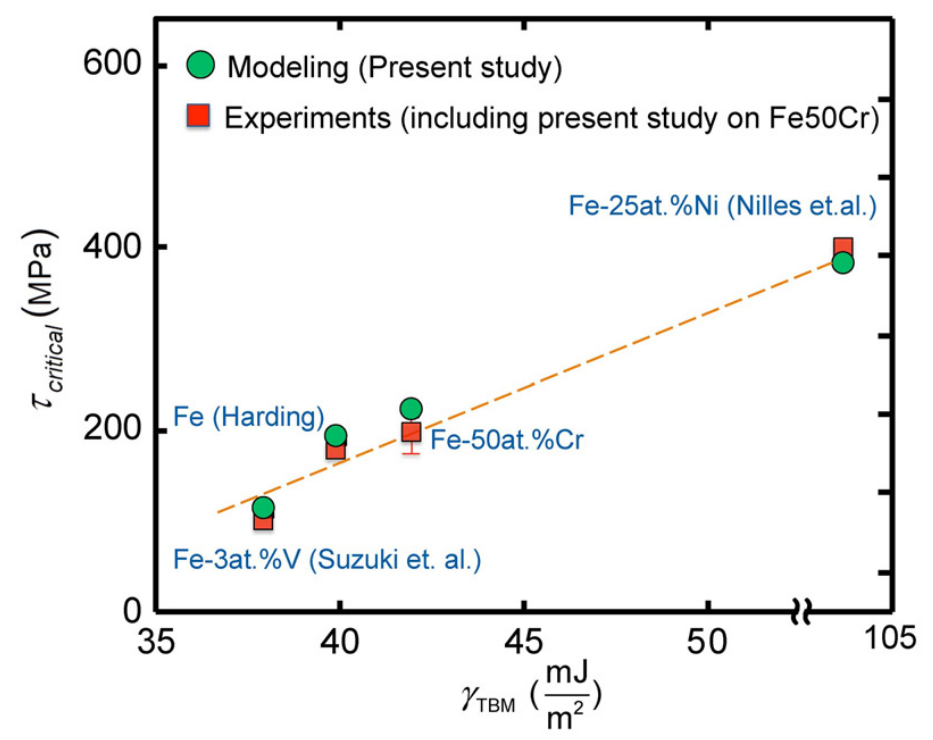

Figure 9. The dependence of the twinning stress on $\gamma_{\mathrm{TBM}}$ for Fe and Fe-based bcc alloys based on the present analysis. Experimentally measured twinning stresses are also presented. Dashed lines are shown to aid the eye.

Table 3. Predicted fault energies of a twin boundary using EAM potential for different metals. The units of fault energies are in $\mathrm{mJ} \mathrm{m}^{-2}$.

\begin{tabular}{llllll}
\hline Metals & $\gamma_{\mathrm{us}}^{\text {twin }}$ & $\gamma_{\mathrm{sf}}$ & $\gamma_{\mathrm{ut}}$ & $2 \gamma_{\mathrm{tsf}}$ & $\gamma_{\mathrm{TBM}}$ \\
\hline Fe (bcc) & 617 & 593 & 628 & 588 & 40 \\
Fe-50 at.\%Cr & 752 & 695 & 759 & 717 & 42 \\
Fe-25 at.\%Ni & 525 & 464 & 549 & 454 & 95 \\
Fe-3 at.\%V & 615 & 546 & 596 & 557 & 39 \\
\hline
\end{tabular}

resistance of the dislocation glide through the crystal with an increase in the Cr content [35]. This contributes to a higher twin nucleation stress in such alloys.

To make our analysis more realistic, we focus on predicting the twin nucleation stress in pure $\mathrm{Fe}$ and an $\mathrm{FeCr}$ alloy with a relatively high $\mathrm{Cr}$ content $(50 \%)$ where the propensity of twinning is higher. Twins have also been observed experimentally in $\mathrm{FeCr}$ alloys with lower concentration of chromium (1\%-3\%) [43]; however, atomistic observations of low $\mathrm{Cr}$ content (11\% and 20\%) $\mathrm{FeCr}$ alloys [35] have shown that slip on the $\left\{\begin{array}{lll}1 & 1 & 0\end{array}\right\}$ planes is primarily the favored deformation mechanism, and it is possible that twins may not be observed in such alloys (except at low temperatures). Throughout this work, we carefully selected the $\mathrm{Fe}-50$ at $\% \mathrm{Cr}$ single-crystal orientations in order to activate multiple twin systems. The experimental observations show that twinning is of paramount importance in the mechanical behavior of $\mathrm{Fe}-50$ at $\% \mathrm{Cr}$ alloys at room temperature since twins nucleate in the early stages of deformation. For example, in [0 10$]$ tensile orientation, twins nucleate in the 'elastic' region of the stress-strain curve, and for [ [ $\left.\begin{array}{llll}1 & 1 & 1\end{array}\right]$ and $\left[\begin{array}{lll}1 & 0 & 1\end{array}\right]$ compressive orientations, twins nucleate approximately below $2 \%$ of the total deformation. 
Table 4. Comparison of twinning stresses obtained through different methods. Present model yields twinning stresses very close to the experimental values.

\begin{tabular}{llll}
\hline $\begin{array}{l}\text { Metals } \\
\text { (bcc) }\end{array}$ & $\begin{array}{l}\tau_{\text {critical }}^{\exp } \\
(\mathrm{MPa})(\text { Experimental) }\end{array}$ & $\begin{array}{l}\tau_{\text {critical }}^{\text {ideal }} \\
(\mathrm{MPa})\end{array}$ & $\begin{array}{l}\tau_{\text {critical }}^{\text {current }} \\
(\mathrm{MPa})\end{array}$ \\
\hline $\mathrm{Fe}$ & $170^{\mathrm{a}}$ & $1530^{\mathrm{b}}$ & 190 \\
$\mathrm{Fe}-50$ at.\%Cr & $203 \pm 13$ & $1600^{\mathrm{b}}$ & 218 \\
$\mathrm{Fe}-25$ at.\%Ni & $398^{\mathrm{c}}$ & $3580^{\mathrm{b}}$ & 377 \\
$\mathrm{Fe}-3$ at.\%V & $90^{\mathrm{d}}$ & $1490^{\mathrm{b}}$ & 109 \\
\hline
\end{tabular}

${ }^{a}$ Harding et al [13,14].

${ }^{\mathrm{b}}$ Approximate calculations based on Ogata et al [10].

${ }^{\mathrm{c}}$ Nilles and Owen [9].

${ }^{\mathrm{d}}$ Suzuki et al [48].

We note that there is no evidence of $\sigma$ phase (spinodal decomposition product) based on extensive TEM analysis (see figure 5). In the present work, the high temperature was selected in the cubic- $\alpha$ (bcc) region with subsequent quenching, and the room temperature microstructure is the $\alpha$ phase in its entirety. The formation of the $\sigma$ phase requires incubation time in the $\sigma$ region of the phase diagram [44-47]. Therefore, our theoretical analysis has been applied to the alloys where the predicted twinning stress is physically consistent with the microstructure. It is worth pointing out that in the case of aging, the $\mathrm{Fe}-50 \% \mathrm{Cr}$ alloys undergo spinodal decomposition [45-47] consisting of Fe- $\left(\alpha_{1}\right)$ and $\mathrm{Cr}-\left(\alpha_{2}\right)$ rich phases, and twins have been observed in such alloys. The twins are believed to originate and reduce the increased lattice friction associated with the $\alpha_{2}$ phase [44], which may eventually cause the $\alpha_{1}$ phase to deform by twinning as well. No aging is undertaken in this study, and hence no compositional gradients are developed.

Twinning is observed to be the only mode of deformation in $\mathrm{Fe}-25$ at. $\% \mathrm{Ni}$ massive martensite at low temperatures and is confined to the $\left\{\begin{array}{lll}1 & 1 & 2\end{array}\right\}$ planes, similar to most bcc metals [9]. Nilles and Owen adopted the bcc pole mechanism [4] to model the twinning stress in $\mathrm{Fe}-25$ at.\%Ni. Accordingly, the authors were able to forward a number of experimentally observed results such as the weak dependence of the twinning stress on the temperature and the effects of the grain size on the twinning stress. The experimentally reported twinning stress by Nilles and Owen for $\mathrm{Fe}-25$ at. $\% \mathrm{Ni}$ is close to the twinning stress predicted by the present theory, as illustrated in table 4. Similarly, the works of Suzuki and Tanino [48] on bcc $\mathrm{Fe}-3$ at.\%V reported the activation of the twins with the highest SF under compression. The twins were observed to nucleate approximately at $90 \mathrm{MPa}$, whereby the growth was assumed to occur through the bcc pole mechanism. With our present analysis, the twinning stress for $\mathrm{Fe}-$ 3 at. $\% \mathrm{~V}$ is predicted to be $109 \mathrm{MPa}$, thus reflecting a close agreement with the experimentally observed value.

The twinnability approach has been forwarded for fcc metals [49] and extended to bcc metals [50]. This approach is useful in explaining the competition between slip and twinning, and the favorability of one over the other. The present analysis can also be used to study the competition between twinning and slip by emphasizing the role of the energy parameters $\gamma_{\text {us }}^{\text {twin }}$ and $\gamma_{\text {твм. }}$. The present calculations (refer to appendix A2) show that twinning is preferred to slip as a deformation mechanism in alloys documented in the present study.

In the present approach, we established an important relation to determine the critical twinning stress in bcc metals. The analysis reveals that the twinning stress at a continuum level can be accurately determined with the combined knowledge of the dislocation interactions 


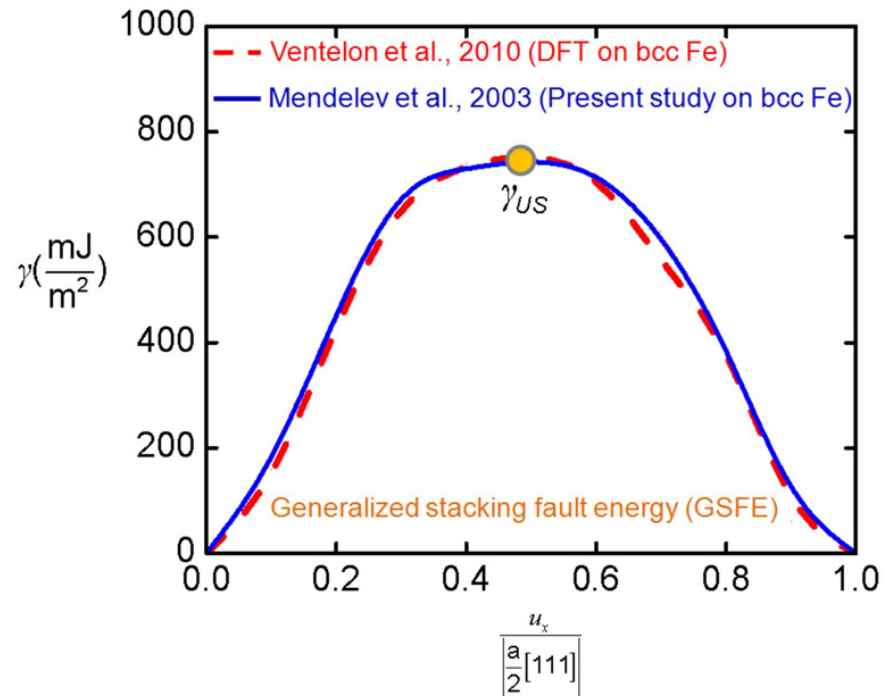

Figure 10. Generalized stacking fault energy (GSFE) of pure bcc Fe obtained through Mendelev et al [23] potential and DFT [41]. The energy profile calculated using the empirical potential is consistent with the one obtained through first principles DFT. The GSFE is calculated by displacing one half of the crystal with respect to the other half by a displacement of $(a / 2)\langle 111\rangle$ on $\{112\}$ plane.

at a mesoscale level and the energy landscape associated with the glide of the dislocations at an atomic level. The knowledge of the energy landscape for deformation twinning, which is quantitatively represented by the GPFE, essentially captures the twin nucleation process in bcc metals. The heterogeneous twin nucleation mechanism incorporated with the energy traversed by the dislocations during twin nucleation process predicts the magnitude of the twinning stress in close agreement with the experimentally measured values.

Figure 10 shows the stacking fault energy values for pure bcc Fe obtained through the Mendelev et al (2003) potential compared to Density functional theory (DFT) [51]. The potentials in the present study extensively utilize results from $a b$ initio calculations and experiments for fitting the properties of alloys [25,28-31]. The accuracy of these potentials for reproducing the thermodynamic properties of the alloys (including properties of the liquid phase and the melting point, defect energies, the interaction energy with the substitutional atoms, etc) is extremely good compared to DFT calculations [25, 28-31]. This further supports that the stacking fault energy values that we predicted for the alloys would be close to the true stacking fault energy values. The ultimate agreement between theory and experiment (within $10 \%$ ) for twinning stress confirms their validity.

\section{Conclusion}

The present analysis reveals that twin nucleation stress in bcc metals depends on the important GPFE parameters $\gamma_{\mathrm{sf}}, \gamma_{\mathrm{ut}}, 2 \gamma_{\mathrm{tsf}}$. We emphasized the importance of GPFE in the present work because it represents the energy barrier that the fractional dislocations must overcome 
to nucleate a twin. To first approximation, we observe that the twin nucleation stresses are higher for alloys with higher $\gamma_{\text {TBM }}$. We note that the overall variation of the GPFE curve with atomic position affects the twinning stress, and thus we validated the need to incorporate the positions of the fractional dislocations in the twinning stress expression. Using elasticity theory, we calculated the interaction energy of the fractional dislocations forming the twin nucleus. GPFE was incorporated into the bcc twinning mechanism to develop an analytical expression to predict the magnitude of the twin nucleation stress, and in the form $\tau_{\text {critical }}=\tau\left(\gamma_{\mathrm{sf}}, \gamma_{\mathrm{ut}}, 2 \gamma_{\mathrm{tsf}}, G, d\right)$. The parameters such as shear modulus $(G)$, the separation distance of the fractional dislocations $d\left(=r_{B}-r_{A}\right)$ and GPFE parameters can all be obtained through atomistic methods, thus excluding the need for any empirical constants. The accuracy of the present analysis was verified by comparing the results to the experimental observations in bcc Fe and Fe-based alloys unveiling excellent agreement.

\section{Acknowledgments}

This work was supported by the National Science Foundation, NSF CMMI-113003. This support is gratefully acknowledged. The authors are grateful to Y Chumlaykov for growing the $\mathrm{Fe}-50$ at.\% $\mathrm{Cr}$ single crystals utilized in the present work.

\section{Appendix A1}

The total energy of the dislocations forming the twin nucleus in figure $1(b)$ can be written as

$$
\begin{aligned}
E_{\text {total }}=-\frac{G b^{2}}{2 \pi} & \left(\ln \left[\frac{r_{B}-r_{A}}{r_{o}}\right]+\ln \left[\frac{r_{B}}{2 r_{o}}\right]+\ln \left[\frac{r_{A}}{r_{o}}\right]\right)+n \alpha G b^{2}-\tau_{\mathrm{RSS}} b\left(r_{A}-r_{o}\right) \\
& -\tau_{\mathrm{RSS}} b\left(r_{B}-2 r_{o}\right)+\left(\int_{0}^{r_{A}} \gamma_{\mathrm{GPFE}} \mathrm{d} x-\int_{0}^{r_{A}} \gamma_{\mathrm{sf}} \mathrm{d} x\right),
\end{aligned}
$$

where the parameters have been previously defined. We minimize the total energy expression (11) with respect to the positions of the dislocations $r_{A}$ and $r_{B}$ on the verge of twinning, and we obtain the following expressions:

$$
\begin{aligned}
& \frac{\partial E_{\text {total }}}{\partial r_{A}}=0 \\
& \frac{\partial E_{\text {total }}}{\partial r_{B}}=0 .
\end{aligned}
$$

Solving equation (12),

$$
-\frac{G b^{2}}{2 \pi}\left(\frac{1}{r_{A}}-\left[\frac{1}{r_{B}-r_{A}}\right]\right)-\tau_{\mathrm{RSS}} b+\gamma_{\mathrm{twin}}^{\prime}=0 .
$$

Equilibrium on dislocation $C$ requires

$$
\frac{G b^{2}}{2 \pi}\left(\frac{1}{r_{B}}+\frac{1}{r_{A}}\right)-\tau_{\mathrm{RSS}} b+\gamma_{\mathrm{twin}}^{\prime}=0
$$

where

$\gamma_{\mathrm{twin}}^{\prime}=\left(\gamma_{\mathrm{ut}}-\frac{\gamma_{\mathrm{ut}}+\gamma_{\mathrm{ssf}}}{2}\right) \sin 2 \pi[2.5-1.21]+\frac{1}{4}\left(2 \gamma_{\mathrm{ut}}-2 \gamma_{\mathrm{tsf}}\right) \sin 2 \pi[N-1.22]$.

For the dislocation configuration in figure $1(b)$, the position $r_{B}$ of dislocation $B$ is calculated from equation (15) under zero external stress $\left(r_{B}=2 r_{A}\right)$ as follows:

$$
r_{B}=\frac{3 G b^{2}}{2 \pi \gamma_{\text {twin }}^{\prime}} \text {. }
$$


However, under an applied stress, the dislocation $A$ in figure $1(b)$ starts to move. Equating equations (14) and (15), we calculate the ratio of the separation distance $r_{A} / r_{B}$ on the verge of twinning $\left(r_{\mathrm{c}}=0\right)$, and obtain the following:

$$
k=\sqrt{3}-1, \quad \text { where } k=\frac{r_{A}}{r_{B}} .
$$

Equation (18) suggests that the third layer (dislocation $C$ ) nucleates when the ratio of the distances of dislocations $A$ and $B\left(r_{A} / r_{B}\right)$ is 0.732 which is consistent with Sleeswyk's (1963) calculation as well. We can rewrite equation (14) by substituting $k$ from equation (18) to obtain the following expression for the critical twinning stress:

$$
\tau_{\text {critical }}=\frac{1}{b}\left\{\gamma_{\mathrm{twin}}^{\prime}-\frac{G b^{2}}{2 \pi}\left(\frac{3-2 \sqrt{3}}{(\sqrt{3}-1) d}\right)\right\},
$$

where

$$
d=r_{B}-r_{A} .
$$

$d$ is the separation distance of the $(a / 6)\left\langle\begin{array}{lll}1 & 1 & 1\end{array}\right\rangle$ dislocations on the verge of twinning, i.e. when dislocation $C$ just starts to move forming a three-layer fault (twin).

Calculations for $\mathrm{Fe}-50 \mathrm{Cr}$

$$
G_{\{112\}\langle 111\rangle}=61 \mathrm{GPa} \text {. }
$$

The position $r_{B}$ of dislocation $B$ is calculated from equations (16)-(17) as

$$
r_{B}=207 \AA \text {. }
$$

From equation (18): $r_{A}=0.732 r_{B}=151 \AA$

$$
d=r_{B}-r_{A}=56 \AA \text {. }
$$

Substituting into equation (19), we obtain

$$
\tau_{\text {critical }}=218(\mathrm{MPa}) \text {. }
$$

The calculated critical stress for twinning in $\mathrm{Fe}-50 \% \mathrm{Cr}$ is in excellent agreement with the experimental value. Our calculations show that the energy contribution to the total twinning stress of $218 \mathrm{MPa}$ in $\mathrm{Fe}-50$ at $\% \mathrm{Cr}$ is predominantly governed by GPFE. In order to nucleate a twin in $\mathrm{Fe}-50$ at $\% \mathrm{Cr}, 65 \%$ of the total stress (approximately $143 \mathrm{MPa}$ ) is invested in overcoming $\gamma^{\prime}$ alone in equation (19). Similar calculations are employed to predict the twinning stresses for the rest of the bcc metals and alloys presented in this work.

\section{Appendix A2}

The twinnability approach has been forwarded for fcc metals [49] and extended to bcc metals [50]. The approach is useful in explaining the competition between slip and twinning, and the favorability of one over the other. Figure A2.1 shows GPFE and GSFE (Generalized Stacking Fault Energy) for Fe-50 at.\%Cr. GSFE is calculated by displacing one-half of the crystal with respect to the other half by a displacement of $(a / 2)\left\langle\begin{array}{lll}1 & 1 & 1\rangle\end{array}\right.$ on the $\left\{\begin{array}{lll}1 & 1 & 2\end{array}\right\}$ plane, and thus represents the energy barrier $\left(\gamma_{\mathrm{us}}\right)$ required to nucleate a full $(a / 2)\langle 111\rangle$ slip dislocation. Li et al [50] have calculated the ratio of $\gamma_{\mathrm{TBM}}$ to $\Delta \gamma_{\mathrm{us}}$ for a number of bcc metals, and depending on the ratio, the authors have shown that twinning is favorable for metals if the ratio is less than one. The reason behind the argument is that twinning requires the emission of subsequent $b_{\mathrm{p}}=(a / 6)\left\langle\begin{array}{lll}1 & 1 & 1\end{array}\right\rangle$ fractional dislocations on consecutive $\left\{\begin{array}{lll}1 & 1 & 2\end{array}\right\}$ planes. An analysis of figure A2.1 shows that once a dislocation $b_{\mathrm{p}}=(a / 6)\langle 111\rangle$ is formed, 


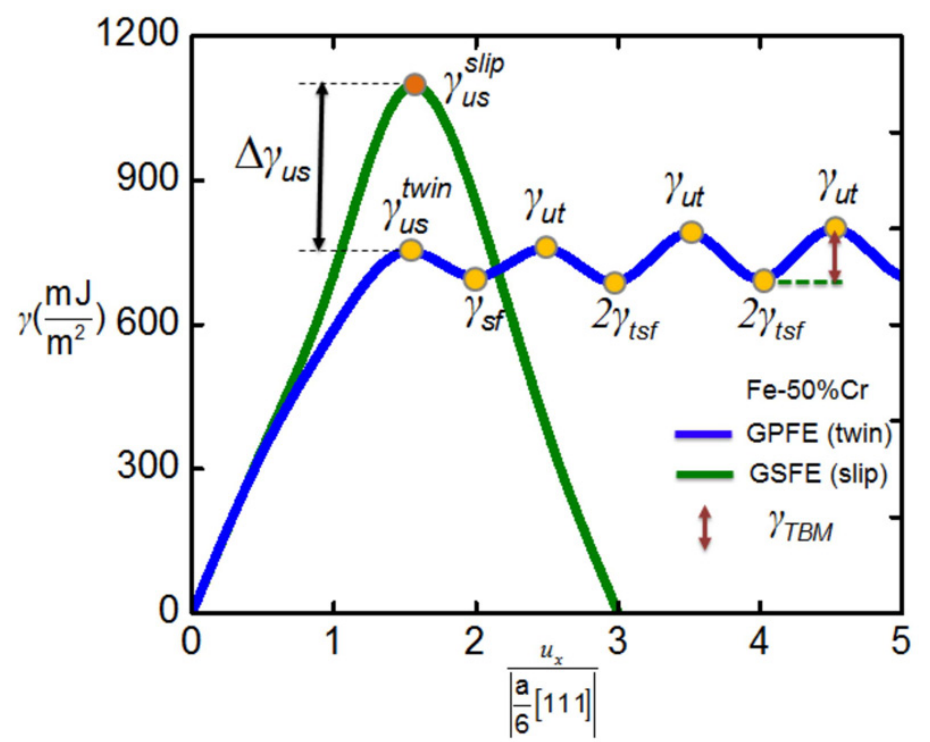

Figure A2.1. The GPFE and the GSFE obtained for $\mathrm{Fe}-50 \% \mathrm{Cr}$ at various normalized shear displacements. The normalized displacements and the stacking fault energies corresponding to slip and twin nucleation are shown. The GPFE represents the energy barrier to nucleate a twin while the GSFE represents the energy barrier to nucleate a slip.

Table A2.1. Energy values corresponding to the GSFE and GPFE for a number of bcc alloys. $\gamma_{\text {us }}$ represents the energy barrier to nucleate a slip while $\gamma_{\text {us }}^{\text {twin }}$ represents the energy barrier to nucleate the first layer stacking fault in the twin. The units of the fault energies are in $\mathrm{mJ} \mathrm{m}^{-2}$.

\begin{tabular}{lrllll}
\hline Metals & \multicolumn{1}{c}{$\gamma_{\text {us }}^{\text {slip }}$} & $\gamma_{\text {us }}^{\text {twin }}$ & $\Delta \gamma_{\text {us }}=\gamma_{\text {us }}^{\text {slip }}-\Delta \gamma_{\text {us }}^{\text {twin }}$ & $\gamma_{\text {TBM }}$ & $\frac{\gamma_{\text {TBM }}}{\Delta \gamma_{\text {us }}}$ \\
\hline $\mathrm{Fe}$ & 752 & 617 & 135 & 40 & 0.30 \\
$\mathrm{Fe}-50 \% \mathrm{Cr}$ & 1060 & 752 & 308 & 42 & 0.14 \\
$\mathrm{Fe}-25 \% \mathrm{Ni}$ & 709 & 525 & 184 & 95 & 0.51 \\
$\mathrm{Fe}-3 \% \mathrm{~V}$ & 741 & 615 & 131 & 39 & 0.29 \\
\hline
\end{tabular}

the competition between emitting a second fractional dislocation or continuing for a full slip dislocation $(a / 2)\langle 1111\rangle$ depends on the energy barrier to emit an $(a / 3)\langle 111\rangle$ dislocation on the same $\left\{\begin{array}{lll}1 & 1 & 2\end{array}\right\}$ plane (represented by $\gamma_{\mathrm{us}}$ ) or the energy to emit a subsequent $b_{\mathrm{p}}=(a / 6)\langle 111\rangle$ dislocations (represented by $\gamma_{\text {TBM }}$ ) on consecutive $\left\{\begin{array}{lll}1 & 1 & 2\end{array}\right\}$ planes. In figure 20 , it can be seen that the magnitude of $\gamma_{\mathrm{TBM}}$ is much lower than $\Delta \gamma_{\mathrm{us}}$ for $\mathrm{Fe}-50 \% \mathrm{Cr}$, yielding the ratio of $\gamma_{\mathrm{TBM}}$ to $\Delta \gamma$ us to be less than $1(0.14)$. Hence, it is easier to emit subsequent $(a / 6)\langle 111\rangle$ dislocations on $\left\{\begin{array}{lll}1 & 1 & 2\end{array}\right\}$ planes to form a twin than to form a full slip dislocation in $\mathrm{Fe}-50 \% \mathrm{Cr}$. Likewise, as presented in table A2.1, an analysis of these energy parameters for all the metals documented in the present study show that the ratio of $\gamma_{\mathrm{TBM}}$ to $\Delta \gamma_{\text {us }}$ is less than 1 , which shows the favorability of twinning over slip. 


\section{References}

[1] Mahajan S and Williams D F 1973 Deformation twinning in metals and alloys Int. Mater. Rev. 1843

[2] Christian J W and Mahajan S 1995 Deformation twinning Prog. Mater. Sci. 391

[3] Meyers M A, Vohringer O and Lubarda V A 2001 The onset of twinning in metals: a constitutive description Acta Mater. 494025

[4] Cottrell A H and Bilby B A 1951 LX. A mechanism for the growth of deformation twins in crystals Phil. Mag. Ser. 42573

[5] Priestner R and Leslie W C 1965 Nucleation of deformation twins at slip plane intersections in B.C.C. metals Phil. Mag. 11895

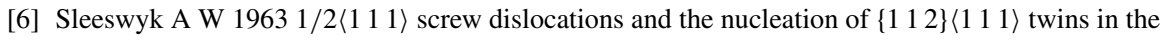
b.c.c. lattice Phil. Mag. 81467

[7] Lagerlof K P D 1993 On deformation twinning in b.c.c. metals Acta Metall. Mater. 412143

[8] Ogawa K 1965 Edge dislocations dissociated in $\left\{\begin{array}{lll}1 & 1 & 2\end{array}\right\}$ planes and twinning mechanism of b.c.c. metals Phil. Mag. 11217

[9] Nilles J L and Owen W S 1972 Deformation twinning of martensite Mechanical Behavior of Materials vol 1 (Kyoto, Japan: The Society of Metals) pp 97-105

[10] Ogata S, Li J and Yip S 2005 Energy landscape of deformation twinning in bcc and fcc metals Phys. Rev. B 71224102

[11] Li S et al 2010 Superelasticity in bcc nanowires by a reversible twinning mechanism Phys. Rev. B 82205435

[12] Ojha A et al 2014 Twin migration in Fe-based bcc crystals: theory and experiments Phil. Mag. $941-25$

[13] Harding J 1967 The yield and fracture behavior of high-purity iron single crystals at high rate crystals at high rates of strain Proc. R. Soc. A 299464

[14] Harding J 1968 The yield and fracture of high-purity iron single crystals under repeated tensile impact loading Mem. Sci. Rev. Met. 65245

[15] Duesbery M S, Vitek V and Bowen D K 1973 The effect of shear stress on the screw dislocation core structure in body-centered cubic lattices Proc. R. Soc. Lond. A 33285

[16] Duesbery M S and Vitek V 1998 Plastic anisotropy in b.c.c. transition metals Acta Mater. 461481

[17] Basinski Z S, Duesbery M S and Taylor R 1970 Screw dislocation in a model sodium lattice Phil. Mag. 211201

[18] Bristowe P D and Kroecker A G 1975 A computer simulation study of the structures of twin boundaries in body-centered cubic crystals Phil. Mag. 31503

[19] Vitek V 1974 Cryst. Latt. Defects 51

[20] Vitek V, Perrin R C and Bowen D K 1970 The core structure of 1/2(111) screw dislocations in b.c.c. crystals Phil. Mag. 211049

[21] Vitek V 1970 Multilayer stacking faults and twins on $\{211\}$ planes in bcc metals Scr. Metall. 4725

[22] Ostapovets A and Paidar V 2007 Planar defects on (1 1 2) in bcc crystals Mater. Sci. Forum 567-568 69

[23] Paidar V 1983 Multilayer stacking faults in b.c.c. metals Phil. Mag. A 48231

[24] Kibey S et al 2007 Predicting twinning stress in fcc metals: linking twin-energy pathways to twin nucleation Acta Mater. 556843

[25] Stukowski et al 2009 Efficient implementation of the concentration-dependent embedded atom method for molecular dynamics and Monte-Carlo simulations Modelling Simul. Mater. Sci. Eng. 7075005

[26] Plimpton S 1995 Fast parallel algorithms for short-range molecular dynamics J. Comput. Phys. 117 1-19

[27] Vitek V Intrinsic stacking faults in body-centred cubic crystals Phil. Mag. 18 773-86

[28] Mendelev M I et al 2007 Simulation of the interaction between Fe impurities and point defects in V Phys. Rev. B 76214105

[29] Mendelev M I et al 2003 Development of new interatomic potentials appropriate for crystalline and liquid iron Phil. Mag. 83 3977-94 
[30] Bonny G, Pasianot R C and Malerba L 2009 Fe-Ni many-body potential for metallurgical applications Modelling Simul. Mater. Sci. Eng. 17025010

[31] Malerba L et al 2010 Ab initio calculations and interatomic potentials for iron and iron alloys: achievements within the perfect project J. Nucl. Mater. 406 7-18

[32] Pietrokowsky P 1966 Lattice parameter of alpha iron by divergent beam diffraction J. Appl. Phys. 374560

[33] Ledbetter H M and Reed R P 1973 Elastic properties of metals and alloys: I. Iron, nickel, and iron-nickel alloys J. Phys. Chem. Ref. Data 2531

[34] Zhang $\mathrm{H}$ et al 2012 Elastic parameters of paramagnetic iron-based alloys from first-principles calculations Phys. Rev. B 85054107

[35] Farkas D et al 1996 Embedded atom computer simulation of lattice distortion and dislocation core structure and mobility in Fe-Cr alloys Acta Mater. 44409

[36] Hirth J P and Lothe J 1982 Theory of Dislocations (New York: Wiley)

[37] Hull D and Bacon D J 1984 Introduction to Dislocations vol 257 (Oxford: Pergamon)

[38] Schoeck G 1994 The generalized Peierls-Nabarro model Phil. Mag. A 69 1085-95

[39] Schoeck G 1998 Deviations from Volterra dislocations Phil. Mag. Lett. 77 141-6

[40] Tadmor E B and Miller R E 2011 Modeling Materials: Continuum, Atomistic and Multiscale Techniques (Cambridge: Cambridge University Press)

[41] Leibfried G and Dietze H-D 1949 Zur theorie der schraubenversetzun Z. Phys. 126 790-808

[42] Patriarca L et al 2012 Twin nucleation and migration in FeCr single crystals Mater. Charact. 75 165

[43] Kelley M J and Stoloff N S 1976 Metall. Trans. A 7331

[44] Mahajan S S Jin and Brasen D 1980 Micro-twinning in a spinodally decomposed $\mathrm{Fe}-\mathrm{Cr}-\mathrm{Co}$ alloy Acta Metall. 28 971-7

[45] Nys T and Gielen P M 1971 Spinodal decomposition in the Fe-Cr system Metall. Trans. 2 1423-8

[46] Dubiel S M and Cieślak J 2011 Sigma-phase in Fe-Cr and Fe-V alloy systems and its physical properties Crit. Rev. Solid State Mater. Sci. 36 191-208

[47] Marcinkowiski M J, Fisher R M and Szirmae A 1964 Effect of $500^{\circ} \mathrm{C}$ aging on the deformation behavior of an iron-chromium alloy Trans. AIME 230 676-89

[48] Suzuki G H, Tanino M and Aoki K 1966 Deformation twin in single crystals of a 3\% vanadium-iron alloy Japan. J. Appl. Phys. 510

[49] Bernstein N and Tadmor E B 2004 Tight-binding calculations of stacking energies and twinnability in fcc metals Phys. Rev. B 69094116

[50] Li S et al 2010 Superelasticity in bcc nanowires by a reversible twinning mechanism Phys. Rev. B 82205435

[51] Ventelon L and Willaime F 2020 Generalized stacking-faults and screw-dislocation core-structure in bcc iron: a comparison between ab initio calculations and empirical potentials Phil. Mag. 90 $1063-74$ 